\title{
The Avian Osteological Collection (Non-Passeriformes) Deposited in the National Museum of Natural History, NAS of Ukraine
}

\section{V. Gorobets}

\author{
National Museum of Natural History, NAS of Ukraine (Kyiv, Ukraine)
}

\begin{abstract}
The Avian Osteological Collection (Non-Passeriformes) Deposited in the National Museum of Natural History, NAS of Ukraine. - Gorobets, L. V. - The article presents the checklist of Non-Passeriformes birds which skeletons are represented in the osteological collection of the National Museum of Natural History, NAS of Ukraine (Kyiv, Ukraine). The dynamics of the collection enrichment and names of collectors are shown. The very first specimens were collected in 1859. The most active work on the collection enrichment was carried out during 1930-1950 by I. G. Pidoplichko, V. O. Antonovich, V. I. Zubareva and others. Even during the World War II the museum's stuff were trying to collect new materials. Bones of birds gained not far from the front line are presented in the collection. The enrichment activities decreased during 1960-1970 and almost totally ceased during 1980-2000. Since 2013, the work has been renewed. To the present day the collection contains bones of 1557 individuals of non-Passerine birds representing 266 wild species and 5 domestic forms. A part of skeletons are remains from museum carcasses: bones of shoulder girdle, sternum, sacrum, shoulder and femoral bones etc. Most individuals were collected in the territory of Ukraine, Russia and the Southern Ocean. A relatively large part of birds was delivered from the Kyiv Zoo.
\end{abstract}

Key words: bird bones, bone collection, osteology.

\section{Introduction}

In the paleontological department of the National Museum of Natural History, NAS of Ukraine (Kyiv, Ukraine) a significant collection of bird bones is deposited. The purpose of the present work is to put in order the collection (during several decades the collection hadn't a special curator) and arrange a checklist of specimens. The Non-Passeriformes are presented here. Compilation of a detailed catalogue of specimens is in process which will include the checklist of all bones and main measurements, and where Passeriformes will be presented.

\section{History of collection}

The earliest label in the collection is dated to 1859 , but relatively regular enrichment has begun at the end of the $19^{\text {th }}$ century. Only $18.4 \%$ of non-Passerine skeletons have labels with that year indicated. However, as long as the collection was enriched by different people, even analysis of $18.4 \%$ of labels allows to monitor the main stages of the collection's creation. During the century there were collectors either pointing out or not the year of the specimen's collection, although data absence on enrichments in some years is not only the year wasn't showed by the collector, but actually an evidence the collection was not enriched. This supposition can be proved by the fact that only 3 labels have the years from 1981-2012. Besides, the museum's stuff confirms that during this time the avian osteological collection was not enriched.

Analysis of the collection enrichment (fig. 1) shows that the most active work was carried out during 1930-1950. There was a break in the collection's enrichment because of the "Soviet stage" of the World War II (1941-1945). However, it has to be specially noted that even at that time museum stuff were trying to collect new materials. For instance, I. Pidoplichko collected a white stork (Ciconia ciconia) in Kyiv on 29 June 1941, when Nazi bombings of the city where started. On 30 November

Corresponding author address: L. V. Gorobets; National Museum of Natural History, NAS of Ukraine; Bohdan Khmelnitsky St. 15, Kyiv, 01030 Ukraine; e-mail: ornitologist@gmail.com 


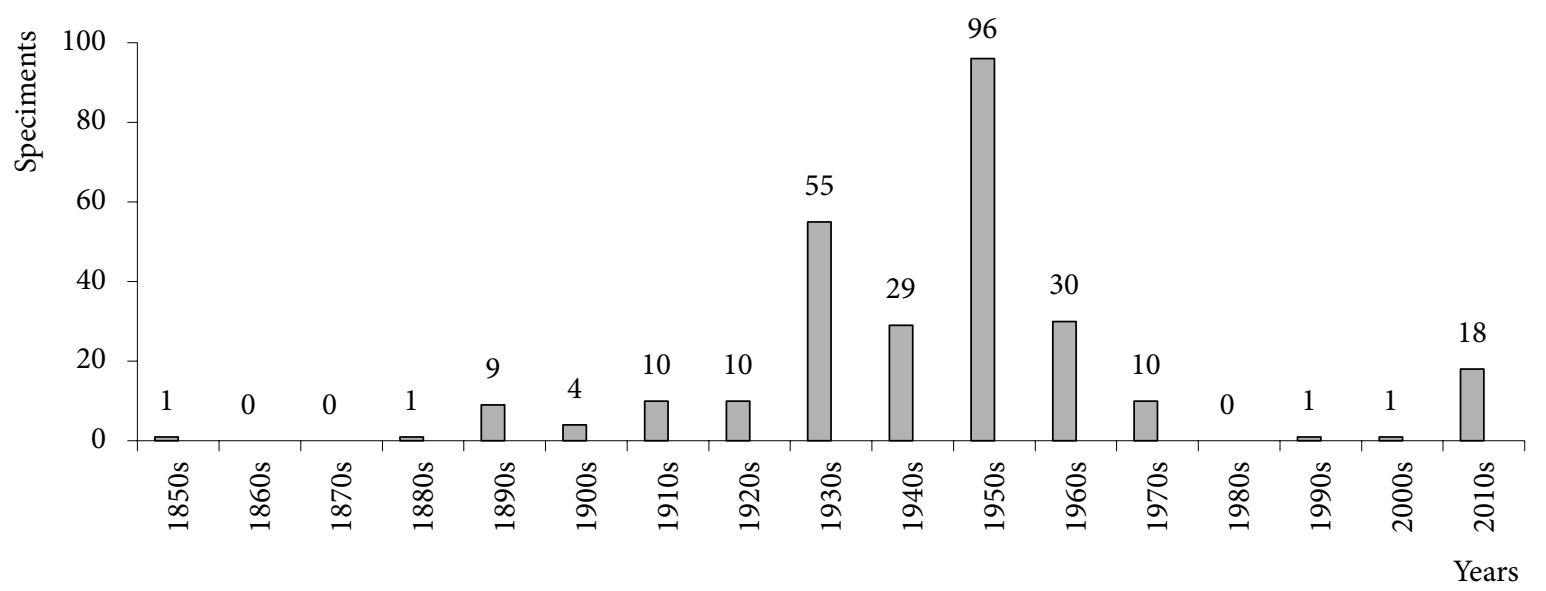

Fig. 1. Dynamics of the avian osteological collection enrichment (based on 275 specimens of wild Non-Passerine bird species, where year is pointed on the label).

Рис. 1. Динаміка надходжень до орнітологічної остеологічної колекції (створено за даними 275 зразків диких не горобиних видів птахів, для яких в етикетці зазначено рік).

1943 V. Zubareva collected an individual of the little owl (Athene noctua) in Kyiv when the city was just released (6 November 1943) and was in ruins.

The enrichment activities noticeably decreased during 1960-1970 and almost totally ceased during 1980-2000. In present days it can't be carried out as actively as in the past because earlier hunting was the main source and now to enrich museum collections only birds dead for reasons irrespective of the collectors are used.

The specimens were collected by: Gerchner (label dated from 1909), O. Lubkin (1911), G. Kotchubej (1912), Svidersky (1913), S. Paramonov (1921), O. Kistiakivskyj (1922), M. Shcherbyna (1926), S. Lubkin (1938), V. Antonovich (1941-1959), I. Pidoplichko (1938-1949), N. Burchak-Abramovich (1939), N. Vereshchagin (1939), Markovsky (1939), B. Popov (1941), Przhebnikov (1941), V. Zubareva (1940-1943), Gristchin (1942), N. Chenyshov (1946), L. Smogorzhevski (1956), O. Vinogradov (1956), D. Borzakovskij (1956-1958), M. Voinstvensky (1957), O. Yatsenja (1957), V. Lagodin (1959), D. Vladyshevsky (1959), M. Shcherbak (1959), N. Kartashov (1959), G. Sapozhnikov (1961), A. Yahontov (1959-1960), A. Tjagunov (1965), O. Gizenko (1967), L. Gorobets (2013 to present).

As of today, the collection contains bones of 1557 individuals of non-Passerine birds belonging to 266 wild species (1475 individuals) and 5 domestic forms (92 individuals). Unfortunately, only a part of skeletons are fully preserved, many of them are remains from museum carcasses: bones of shoulder girdle, sternum, sacrum, shoulder and femoral bones, also proximal parts of ulnar bones and tibiotarsi.

Most individuals were collected in the territory of Ukraine, the Russian Far East (mainly the Khabarovsk Krai), the Komi Republic (North of the European part of Russia) and the Southern Ocean. A relatively large part of birds was delivered from the Kyiv Zoo.

\section{Checklist of species}

The consecution and names of orders and families are given according to Zelenkov and Kurochkin (Зеленков, Курочкин, 2015), and the scientific names of genera and species according to Howard and Moore (Howard, Moore, 2013). For each species the number of individuals (abbreviation: ind.) is indicated as well. 


\section{Order Struthioniformes Latham, 1970}

Genus Struthio Linnaeus, 1758

S. camelus Linnaeus, 1758: 1 ind.

\section{Order Anseriformes Wagler, 1831}

\section{Family Anatidae Vigors, 1825}

Genus Cygnus Garsault, 1764

C. olor (Gmelin, 1789): 14 ind.;

C. atratus (Latham, 1790): 1 ind.;

C. melancoryphus (Molina, 1782): 2 ind.;

C. cygnus (Linnaeus, 1758): 6 ind.;

C. bewickii (Yarrell 1830): 3 ind. Genus Anser Linnaeus, 1758

A. fabalis (Latham, 1787): 5 ind.;

A. albifrons (Scopoli, 1769): 4 ind.;

A. erythropus (Linnaeus, 1758): 7 ind.;

Anser anser (Linnaeus, 1758): 4 ind.;

A. anser domesticus (Linnaeus, 1758): 15 ind. Genus Chen Boie, 1822

C. caerulescens (Linnaeus, 1758): 2 ind. Genus Branta Scopoli, 1769

B. bernicla (Linnaeus, 1758): 2 ind.;

B. ruficollis (Pallas, 1769): 7 ind. Genus Alopochen Stejneger, 1885

A. aegyptiacus (Linnaeus, 1766): 1 ind. Genus Tadorna Boie, 1822

T. ferruginea (Pallas, 1764): 8 ind.;

T. tadorna (Linnaeus, 1758): 9 ind. Genus Cairina Fleming, 1822

C. moschata (Linnaeus, 1758): 2 ind. Genus Aix Boie, 1822

A. galericulata (Linnaeus, 1758): 1 ind. Genus Mareca Stephens, 1824

M. penelope (Linnaeus, 1758): 5 ind.;

M. strepera (Linnaeus, 1758): 4 ind.

Genus Spatula Boie, 1822

S. querquedula (Linnaeus, 1758): 8 ind.;

S. clypeata (Linnaeus, 1758): 6 ind.

Genus Anas Linnaeus, 1758

A. crecca Linnaeus, 1758: 3 ind.;

A. platyrhynchos Linnaeus, 1758: 20 ind.;

A. platyrhynchos domesticus Linnaeus, 1758: 14 ind.;

A. acuta Linnaeus, 1758: 6 ind.

Genus Netta Kaup, 1829

N. rufina (Pallas, 1773): 3 ind.

Genus Aythya F. Boie, 1822

A. ferina (Linnaeus, 1758): 8 ind.;

A. nyroca (Güldenstädt, 1770): 3 ind.;

A. fuligula (Linnaeus, 1758): 1 ind.;
A. marila (Linnaeus, 1758): 4 ind.

Genus Somateria Leach, 1819

S. mollissima (Linnaeus, 1758): 3 ind. Genus Clangula Leach, 1819

C. hyemalis (Linnaeus, 1758): 13 ind. Genus Mellanitta Boie, 1822

M. nigra (Linnaeus, 1758): 1 ind.;

M. fusca (Linnaeus, 1758): 1 ind. Genus Bucephala Baird, 1858

B. clangula (Linnaeus, 1758): 5 ind. Genus Mergellus Selby, 1840

M. albellus (Linnaeus, 1758): 4 ind.

Genus Mergus Linnaeus, 1758

M. serrator Linnaeus, 1758: 6 ind.;

M. merganser Linnaeus, 1758: 3 ind.

\section{Order Galliformes Temminck, 1820}

Family Numididae Reichenbach, 1850

Genus Numida Linnaeus, 1758

N. meleagris (Linnaeus, 1758): 1 ind. Genus Colinus Goldfuss, 1820

C. virginianus (Linnaeus, 1758): 1 ind.

Family Phasianidae Vigors, 1825

Genus Rollulus Bonnaterre, 1791

R. rouloul (Scopoli, 1786): 1 ind.

Genus Meleagris Linnaeus, 1758

M. gallopavo domesticus Linnaeus, 1758: 3 ind. Genus Dendragapus Elliot, 1864

D. falcipennis (Hartlaub, 1855): 1 ind.

Genus Lagopus Brisson, 1760

L. lagopus (Linnaeus, 1758): 29 ind.;

L. mutus (Montin, 1781): 4 ind. Genus Tetrao Linnaeus, 1758

T. mlokosiewiczi Taczanowski, 1875: 3 ind.;

T. tetrix (Linnaeus, 1758): 10 ind.;

T. urogallus Linnaeus, 1758: 9 ind. Genus Bonasa Stephens, 1819

B. bonasia (Linnaeus, 1758): 19 ind. Genus Ammoperdix Gould, 1851

A. griseogularis Brandt, 1843: 2 ind. Genus Tetraogallus J. E. Gray, 1832

T. himalayensis J. E. Gray, 1843: 1 ind. Genus Alectoris Kaup, 1829

A. graeca (Meisner, 1804): 3 ind. Genus Francolinus Stephens, 1819

F. francolinus (Linnaeus, 1758): 4 ind. Genus Perdix Brisson, 1760

P. perdix (Linnaeus, 1758): 18 ind.;

P. daurica (Pallas, 1811): 2 ind. 
Genus Coturnix Garsault, 1764

C. coturnix (Linnaeus, 1758): 8 ind.

Genus Gallus Brisson, 1760

G. gallus (Linnaeus, 1758): 1 ind.;

G. gallus domestica (outbreed): 24 ind.;

G. gallus domestica (short-tail "breed"): 2 ind.;

G. gallus domestica (breed Korol'ok): 1 ind.;

G. gallus domestica (breed Rhode Island Red): 2 ind.;

G. gallus domestica (crested): 1 ind.;

G.gallus $0^{\top} \times$ Numida meleagris $\circ$ : 1 ind.

Genus Phasianus Linnaeus, 1758

Ph. colchicus Linnaeus, 1758: 11 ind.

Genus Chrysolophus J. E. Gray, 1834

Ch. pictus (Linnaeus, 1758): 4 ind.;

Ch. amherstiae Leadbeater, 1829: 1 ind.

Genus Pavo Linnaeus, 1758

P. cristatus Linnaeus, 1758: 2 ind.

\section{Order Phoenicopteriformes Fürbringer, 1888}

Family Phoenicopteridae Bonaparte, 1831

Genus Phoenicopterus Linnaeus, 1758

$P h$. ruber Linnaeus, 1758: 3 ind.;

Ph. chilensis Molina, 1782: 1 ind.

\section{Order Podicipediformes Fürbringer, 1888}

Family Podicipedidae Bonaparte, 1831

Genus Tachybaptus L. Reichenbach, 1853

T. ruficollis (Pallas, 1764): 1 ind.

Genus Podiceps Latham, 1787

P. cristatus (Linnaeus, 1758): 14 ind.;

P. grisegena (Boddaert, 1783): 6 ind.;

P. nigricollis Brehm, 1831: 2 ind.

\section{Order Gaviiformes Coues, 1903}

\section{Family Gaviidae Allen, 1897}

Genus Gavia Forster, 1788

G. stellata (Pontoppidan, 1763): 2 ind.;

G. arctica (Linnaeus, 1758): 6 ind.

\section{Order Sphenisciformes Huxley, 1867}

Family Spheniscidae Bonaparte, 1831

Genus Aptenodytes Miller, 1778

A. forsteri Gray, 1844: 1 ind.

Genus Pygoscelis Wagler, 1832

P. adeliae (Hombron et Jacquinot, 1841): 13 ind.;

P. antarctica (Forster, 1781): 1 ind.

Genus Eudyptes Vieillot, 1816

E. chrysolophus (Brandt, 1837): 2 ind.

\section{Order Procellariiformes Fürbringer, 1888}

Family Diomedeidae Gray, 1840

Genus Diomedea Linnaeus, 1758
D. exulans Linnaeus, 1758: 5 ind.;

D. melanophrys (Temminck 1828): 6 ind.

Genus Phoebetria L. Reichenbach, 1853

Ph. palpebrata (Forster, 1785): 5 ind.

Family Procellariidae Leach, 1920

Genus Macronectes Richmond, 1905

M. giganteus (Gmelin, 1789): 6 ind.

Genus Fulmarus Stephens, 1826

F. glacialoides (Smith, 1840): 3 ind.

Genus Thalassoica L. Reichenbach, 1853

T. antarctica (J. F. Gmelin 1789): 3 ind.

Genus Daption Stephens, 1826

D. capense (Linnaeus, 1758): 11 ind.

Genus Pagodroma Bonaparte, 1856

P. nivea (G. Forster, 1777): 4 ind.

Genus Halobaena Bonaparte, 1856

H. caerulea (Gmelin 1789): 2 ind.

Genus Procellaria Linnaeus, 1758

P. aequinoctialis Linnaeus, 1758: 5 ind.

Genus Puffinus Brisson, 1760

P. grisseus (Gmelin, 1789): 1 ind.;

P. tenuirostris (Temminck, 1836): 3 ind.;

P. puffinus (Brünnich, 1764): 5 ind.

Family Hydrobattidae Mathews, 1912

Genus Oceanodroma L. Reichenbach, 1853

O. monorhis (Swinhoe, 1867): 7 ind.

\section{Order Pelecaniformes Sharpe, 1891}

Family Pelecanidae Rafinesque, 1815

Genus Pelecanus Linnaeus, 1758

P. onoctrotalus Linnaeus, 1758: 4 ind.;

P. crispus Bruch, 1832: 2 ind.

Family Fregatidae Degland et Gerbe, 1867

Genus Fregata Lacépède, 1799

F. minor (Gmelin, 1789): 1 ind.

Family Phalacrocoracidae Reichenbach, 1849

Genus Phalacrocorax Brisson, 1760

Ph. carbo (Linnaeus, 1758): 8 ind.;

Ph. aristotelis (Linnaeus, 1761): 4 ind.;

Ph. atriceps (King, 1828): 10 ind.;

Ph. pygmeus (Pallas, 1773): 3 ind.

\section{Order Ardeiformes Wagler, 1830}

Family Ardeidae Leach, 1820

Genus Ardea Linnaeus, 1758

A. cinerea Linnaeus, 1758: 13 ind.;

A. purpurea (Linnaeus, 1766): 4 ind.

Genus Casmerodius Gloger, 1842

C. albus (Linnaeus, 1758): 4 ind. 
Genus Egretta T. Forster, 1817

E. garzetta (Linnaeus, 1766): 8 ind.

Genus Ardeola F. Boie, 1822

A. ralloides (Scopoli, 1769): 9 ind.

Genus Nycticorax T. Forster, 1817

N. nycticorax (Linnaeus, 1758): 5 ind.

Genus Cochlearius Brisson, 1760

C. cochlearius Linnaeus, 1766 (1 ind.)

Genus Ixobrychus Billberg, 1828

I. minutus (Linnaeus, 1766): 5 ind.

Genus Botaurus Stephens, 1819

B. stellaris (Linnaeus, 1758): 8 ind.

Genus Plegadis Kaup, 1829

P. falcinellus (Linnaeus, 1766): 6 ind.

Genus Platalea Linnaeus, 1758

P. leucordia Linnaeus, 1758: 4 ind.

\section{Order Ciconiiformes Bonaparte, 1854}

Family Ciconiidae Sundevall, 1836

Genus Anastomus Bonnaterre, 1791

A. oscitans (Boddaert, 1783): 1 ind.

Genus Ciconia Brisson, 1760

C. nigra (Linnaeus, 1758): 4 ind.;

C. ciconia (Linnaeus, 1758): 8 ind.

Genus Leptoptilos Lesson, 1831

L. crumeniferus (Lesson, 1831): 1 ind.

Order Gruiformes Bonaparte, 1854

Family Gruidae Vigors, 1825

Genus Grus Brisson, 1760

G. grus (Linnaeus, 1758): 4 ind.;

G. leucogeranus Pallas, 1773:1 ind.

Genus Anthropoides Vieillot, 1816

A. virgo (Linnaeus, 1758): 7 ind.

Genus Balearica Brisson, 1760

B. pavonina (Linnaeus, 1758): 3 ind.

Family Rallidae Rafinesque, 1815

Genus Rallus Linnaeus, 1758

R. aquaticus Linnaeus, 1758: 3 ind.

Genus Crex Bechstein, 1803

C. crex (Linnaeus, 1758): 7 ind.

Genus Porzana Vieillot, 1816

P. porzana (Linnaeus, 1766): 7 ind.

Genus Zapornia Leach, 1816

Z. parva (Scopoli, 1769): 6 ind.

Genus Gallinula Brisson, 1760

G. chloropus (Linnaeus, 1758): 8 ind.

Genus Porphyrio Brisson, 1760

P. porphyrio (Linnaeus, 1758): 3 ind.
Genus Fulica Linnaeus, 1758

F. atra Linnaeus, 1758: 16 ind.

Order Charadriiformes Huxley, 1867

Family Burhinidae Mathews, 1912

Genus Burhinus

B. oedichemus: 3 ind.

Family Glareolidae C.L. Brehm, 1831

Genus Glareola Brisson, 1760

G. pratincola Linnaeus, 1766: 4 ind.;

G. nordmanni Fischer von Waldheim, 1842: 1 ind.

Family Haematopodidae Bonaparte, 1838

Genus Haematopus Linnaeus, 1758

H. ostralegus Linnaeus, 1758: 6 ind.

Family Recurvirostridae Bonaparte, 1831

Genus Himantopus Brisson, 1760

H. himantopus (Linnaeus, 1758): 3 ind.

Genus Recurvirostra Linnaeus, 1758

R. avosetta Linnaeus, 1758: 8 ind.

Family Charadriidae Leach, 1820

Genus Vanellus Brisson, 1760

V. vanellus (Linnaeus, 1758):12 ind.

Genus Pluvialis Brisson, 1760

P. apricaria (Linnaeus, 1758): 4 ind.;

P. squatarola (Linnaeus, 1758): 2 ind.

Genus Charadrius Linnaeus, 1758

Ch. hiaticula Linnaeus, 1758:5 ind.;

Ch. dubius Scopoli, 1786: 3 ind.;

Ch. alexandrinus Linnaeus, 1758: 5 ind.;

Ch. asiaticus (Pallas, 1773): 1 ind.

Family Scolopacidae Rafinesque, 1815

Genus Limosa Brisson, 1760

L. limosa (Linnaeus, 1758): 2 ind.

Genus Numenius Brisson, 1760

N. phaeopus (Linnaeus, 1758): 1 ind.;

N. arquata (Linnaeus, 1758): 6 ind.

Genus Tringa Linnaeus, 1758

T. erythropus (Pallas, 1764): 3 ind.;

T. totanus (Linnaeus, 1758): 11 ind.;

T. stagnatilis (Bechstein, 1803): 1 ind.;

T. nebularia (Gunnerus, 1767): 8 ind.;

T. ochropus Linnaeus, 1758: 5 ind.;

T. glareola (Linnaeus, 1758): 10 ind.

Genus Xenus Kaup, 1829

$X$. cinereus (Güldenstädt, 1775): 2 ind.

Genus Actitis Illiger, 1811

A. hypoleucos (Linnaeus, 1758): 3 ind. 
Genus Arenaria Brisson, 1760

A. interpres (Linnaeus, 1758): 1 ind.

Genus Phalaropus Brisson, 1760

Ph. lobatus (Linnaeus, 1758): 1 ind.

Genus Scolopax Linnaeus, 1758

S. rusticola Linnaeus, 1758: 13 ind. Genus Gallinago Brisson, 1760

G. solitaria Hodgson, 1831: 2 ind.;

G. stenura (Bonaparte, 1831): 1 ind.;

G. megala Swinhoe, 1861: 1 ind.;

G. media (Latham, 1787): 4 ind.;

G. gallinago (Linnaeus, 1758): 6 ind.

Genus Lymnocryptes F. Boie, 1826

L. minimus (Brunnich, 1764): 1 ind.

Genus Calidris Merrem, 1804

C. alba Pallas, 1764: 3 ind.;

C. minuta (Leisler, 1812): 1 ind.;

C. temminckii (Leisler, 1812): 1 ind.;

C. subminuta Middendorff, 1853: 1 ind.;

C. alpine (Linnaeus, 1758): 4 ind.;

C. ferruginea (Pontoppidan, 1763): 1 ind. Genus Limicola Koch, 1816

L. falcinellus (Pontoppidan, 1763): 4 ind. Genus Philomachus Merrem, 1804

Ph. pugnax (Linnaeus, 1758): 11 ind.

Family Alcidae Leach, 1820

Genus Alca Linnaeus, 1758

A. torda Linnaeus, 1758: 2 ind.

Genus Uria Brisson, 1760

$U$. aalge (Pontoppidan, 1763): 2 ind.

Genus Cyclorrhynchus Kaup, 1829

C. psittacula (Pallas, 1769): 1 ind.

Genus Fratercula Brisson, 1760

F. arctica (Linnaeus, 1758): 2 ind.;

F. cirrhata (Pallas, 1769): 2 ind.

\section{Family Laridae Rafinesque, 1815}

Genus Catharacta Brünnich, 1764

C. antarctica (Lesson, 1831): 3 ind.

Genus Stercorarius Brisson, 1760

S. parasiticus (Linnaeus, 1758): 2 ind.;

S. longicaudus Vieillot, 1819: 1 ind.

Genus Larus Linnaeus, 1758

L. canus Linnaeus, 1758: 3 ind.;

L. argentatus Pontoppidan, 1763: 1 ind.;

L. argentatus/cachinnans: 6 ind.;

L. fuscus Linnaeus, 1758: 4 ind.;

L. dominicanus Lichtenstein, 1823: 6 ind.;

L. marinus Linnaeus, 1758: 1 ind.;

L. ichthyaetus (Pallas, 1773): 2 ind.;
L. ridibundus Linnaeus, 1766: 9 ind.;

L. melanocephalus Temminck, 1820: 4 ind.;

L. genei Brème, 1839: 4 ind.;

L. minutus Pallas, 1776: 1 ind. Genus Rissa Stephens, 1826

R. tridactyla (Linnaeus, 1758): 2 ind.

Genus Chlidonias Rafinesque, 1822

Ch. leucopterus (Temminck, 1815): 4 ind.;

Ch. niger (Linnaeus, 1758): 6 ind.

Genus Sterna Linnaeus, 1758

S. nilotica Gmelin, 1789: 6 ind.;

S. caspia Pallas, 1770: 2 ind.;

S. hirundo Linnaeus, 1758: 10 ind.;

S. paradisaea Pontoppidan, 1763: 13 ind.;

S. albifrons Pallas, 1764: 5 ind.;

S. sandvicensis Latham, 1787: 1 ind.

Genus Cerorhinca Bonaparte, 1828

C. monocerata Pallas, 1811: 1 ind.

Genus Syrrhaptes Illiger, 1811

S. paradoxus (Pallas, 1773): 4 ind. Genus Pterocles Temminck, 1815

P. orientalis (Linnaeus, 1758): 1 ind.

\section{Order Otidiformes Wagler, 1830}

Family Otididae Rafinesque, 1815

Genus Tetrax T. Forster, 1817

T. tetrax (Linnaeus, 1758): 2 ind. Genus Otis Linnaeus, 1758

O. tarda Linnaeus, 1758: 7 ind.

Genus Chlamydotis Lesson, 1839

Ch. undulata (Jacquin, 1784): 2 ind.

\section{Order Columbiformes Latham, 1790}

Family Columbidae Leach, 1820

Genus Columba Linnaeus, 1758

C. livia Gmelin, 1789: 4 ind.;

C. livia domestica Gmelin, 1789: 11 ind.;

C. oenas Linnaeus, 1758: 5 ind.;

C. palumbus Linnaeus, 1758: 3 ind.;

C. cayennensis Bonnaterre, 1792: 1 ind.

Genus Streptopelia Bonaparte, 1855

S. turtur (Linnaeus, 1758): 9 ind.;

S. orientalis (Latham, 1790): 6 ind.;

S. decaocto (Frivaldszky, 1838): 8 ind.;

S. risoria (Linnaeus, 1758): 1 ind.;

S. senegalensis (Linnaeus, 1766): 4 ind. Genus Chalcophaps Gould, 1843

Ch. indica (Linnaeus, 1758): 1 ind.

Genus Goura Stephens, 1819

G. cristata (Pallas, 1764): 1 ind. 


\section{Order Cuculiformes Wagler, 1830}

Family Cuculidae Leach, 1820

Genus Cuculus Linnaeus, 1758

C. canorus (Linnaeus, 1758): 11 ind.

Order Caprimulgiformes Ridgway, 1881

\section{Family Caprimulgidae Vigors, 1825}

Genus Caprimulgus Linnaeus, 1758

C. indicus (Latham, 1790): 1 ind.;

C. europaeus Linnaeus, 1758: 12 ind.

\section{Order Apodiformes Peters, 1940}

Family Apodidae Olphe-Gailliard, 1887

Genus Apus Scopoli, 1777

A. melba (Linnaeus, 1758): 1 ind.

A. apus (Linnaeus, 1758): 11 ind.

\section{Order Strigiformes Wagler, 1830}

Family Tytonidae Mathews, 1912

Genus Tyto Billberg, 1828

T. alba (Scopoli, 1769): 2 ind.

Family Strigidae Leach, 1820

Genus Otus Pennant, 1769

O. scops (Linnaeus, 1758): 2 ind.

Genus Bubo Duméril, 1805

B. bubo (Linnaeus, 1758): 4 ind.

Genus Nyctea Stephens, 1826

N. scandiaca (Linnaeus, 1758): 1 ind.

Genus Athene F. Boie, 1822

A. noctua (Scopoli, 1769): 4 ind.

\section{Order Accipitriformes Vieillot, 1816}

Family Pandionidae Bonaparte, 1854

Genus Pandion Savigny, 1809

P. haliaetus (Linnaeus, 1758): 8 ind.

Family Accipitridae Vigors, 1824

Genus Pernis Cuvier, 1816

P. apivorus (Linnaeus, 1758): 11 ind.

Genus Milvus Lacépède, 1799

M. migrans (Boddaert, 1783): 15 ind.

Genus Haliaeetus Savigny, 1809

H. albicilla (Linnaeus, 1758): 15 ind.

Genus Gypaetus Storr, 1784

G. barbatus (Linnaeus, 1758): 1 ind.

Genus Neophron Savigny, 1809

N. percnopterus (Linnaeus, 1758): 1 ind.

Genus Gyps Savigny, 1809

G. fulvus (Hablizl, 1783): 2 ind.

Genus Aegypius Savigny, 1809

Ae. monachus (Linnaeus, 1766): 3 ind.
Genus Circaetus Vieillot, 1816

C. gallicus (Gmelin, 1788): 7 ind.

Genus Circus Lacépède, 1799

C. cyaneus (Linnaeus, 1766): 6 ind.;

C. macrourus S. G. Gmelin, 1770: 7 ind.;

C. pygargus (Linnaeus, 1758): 7 ind.;

C. aeruginosus (Linnaeus, 1758): 13 ind.

Genus Accipiter Brisson, 1760

A. nisus (Linnaeus, 1758): 12 ind.;

A. gentilis (Linnaeus, 1758): 20 ind.

Genus Buteo Lacépède, 1799

B. buteo (Linnaeus, 1758): 11 ind.;

B. buteo vulpinus: 1 ind.;

B. hemilasius Temminck \& Schlegel, 1844: 1ind.;

B. ruffinus (Cretzschmar, 1829): 1 ind.;

B. lagopus (Pontoppidan, 1763): 13 ind.

Genus Aquila Brisson, 1760

A. pomarina C.L. Brehm, 1831: 3 ind.;

A. clanga Pallas, 1811: 3 ind.;

A. rapax (Temminck, 1828): 6 ind.;

A. heliaca Savigny, 1809: 3 ind.;

A. chrysaetos (Linnaeus, 1758): 9 ind.

Genus Hieraaetus Kaup, 1844

H. pennatus (Gmelin, 1788): 1 ind.

Order Falconiformes Sharpe, 1874

Family Falconidae Leach, 1820

Genus Falco Linnaeus, 1758

F. naumanni Fleischer, 1818: 2 ind.;

F. tinnunculus Linnaeus, 1758: 19 ind.;

F. vespertinus Linnaeus, 1766: 5 ind.;

F. columbarius Linnaeus, 1758: 4 ind.;

F. subbuteo Linnaeus, 1758: 4 ind.;

F. cherrug Gray, 1834: 6 ind.;

F. peregrinus Tunstall, 1771: 5 ind.

Order Psitaciformes Wagler, 1830

Family Psittaculidae Rafinesque, 1815

Genus Melopsittacus Gould, 1840

M. undulatus (Shaw, 1805): 6 ind.

Genus Agapornis Selby, 1836

A. personata Reichenow, 1887: 1 ind.

Genus Hapalopsittaca Ridgway, 1912

H. amazonina (Des Murs, 1845): 1 ind.

Order Colliformes Murie, 1872

Genus Corythaixoides A. Smith, 1833

C. leucogaster (Rüppell, 1842): 1 ind.

Order Bucerotiformes Fürbringer, 1888

Family Upupidae Leach, 1820 
Genus Upupa Linnaeus, 1758

U. epops Linnaeus, 1758: 8 ind.

\section{Order Piciformes Linnaeus, 1758}

Family Rhamphastidae Vigors, 1825

Genus Ramphastos Linnaeus, 1758

R. toco Statius Müller, 1776: 1 ind.

Family Picidae Leach, 1820

Genus Jynx Linnaeus, 1758

J. torquilla Linnaeus, 1758: 6 ind.

Genus Dendrocopos Koch, 1816

D. minor (Linnaeus, 1758): 3 ind.;

D. leucotos (Bechstein, 1802): 5 ind.;

D. medius: 5 ind.;

D. syriacus (Hemprich et Ehrenberg, 1833): 1 ind.;

D. major (Linnaeus, 1758): 22 ind.

Genus Picoides Lacépède, 1799

P. tridactylus (Linnaeus, 1758): 3 ind.
Genus Dryocopus F. Boie, 1826

D. martius (Linnaeus, 1758): 11 ind.

Genus Picus Linnaeus, 1758

P. canus Gmelin, 1788: 7 ind.;

P. viridis Linnaeus, 1758: 2 ind.

Order Coraciiformes Forbes, 1884

Family Coraciidae Rafinesque, 1815

Genus Coracias Linnaeus, 1758

C. garrulus Linnaeus, 1758: 11 ind.

Family Meropidae Rafinesque, 1815

Genus Merops Linnaeus, 1758

M. apiaster Linnaeus, 1758: 8 ind.;

M. superciliosus Linnaeus, 1766: 1 ind.

Family Alcedinidae Rafinesque, 1815

Genus Alcedo Linnaeus, 1758

A. athis Linnaeus, 1758: 5 ind.

\section{Conclussion}

The species diversity of avian osteological collection deposited in the National Museum of Natural History, NAS of Ukraine is relatively large. However, regional restrictions of the collection's application is noticeable. It can be used in research of the Quaternary fauna of East Europe. Species identification of remains from other regions may be complicated owing to absence of the same taxons. Many species are represented by several individuals and it is an essential advantage of the collection.

\section{References}

Зеленков, Н. В., Курочкин, Е. Н. Класс Aves // Ископаемые позвоночные России и сопредельных стран. Ископаемые рептилии и птицы. Часть 3. - Москва : ГЕОС, 2015. - С. 86-109. [Zelenkov, N. V., Kurochkin, E. N. Class Aves // Fossil vertebrates of Russia and adjacent countries. Fossil Reptiles and Birds. Part 3. - Moscow: GEOS, 2015. - P. 86-109. (in Russian).]

Howard, R., Moore, A. The Howard and Moore Complete Checklist of the Birds of the World. 4th edn. Vol. 1: Non-passerines. - Eastbourne: Aves Press, 2013. - 461 p.

Орнітологічна остеологічна колекція (Негоробині птахи) Національного науково-природничого музею НАН України. - Горобець, Л. В. - В статті наведено перелік видів негоробиних птахів, скелети яких наявні в остеологічній колекції Національного науково-природничого музею НАН України (Київ, Україна). Наведено динаміку поповнення та прізвища колекторів. Перші екземпляри були добуті в 1859 р. Найбільш активно робота з поповнення тривала в 1930-1950 роках, зусиллями І. Г. Підоплічка, В. О. Антоновича, В. І. Зубарєвої та інших. Навіть в часи Другої світової війни працівники музею намагались поповнювати колекцію. Наявні скелети птахів добуті в безпосередній близькості від фронту. У 1960-1970 рр. активність поповнення зменшилася і майже повністю припинилася в 1980-2000. 32013 року відновились роботи з поповнення колекції. На сьогодні в колекції наявні кістки 1557 особин негоробиних птахів, які належать до 266 диких видів та 5 одомашнених форм. Значна частина особин представлена кістками, які залишились після виготовлення музейних тушок: плечові, стегнові, пояси кінцівок тощо. Переважна більшість зразків добуто на території України, Росії та Південного океану. Значна частина птахів надійшла із Київського зоопарку.

Ключові слова: кістки птахів, колекція кісток, остеологія.

Адреса для зв’язку: Л. В. Горобець; Національний науково-природничий музей НАН України, вул. Богдана Хмельницького 15, м. Київ, 01030 Україна; e-mail: ornitologist@gmail.com 